\title{
Genome-wide association analyses of North American Rheumatoid Arthritis Consortium and Framingham Heart Study data utilizing genome-wide linkage results
}

\author{
Yun Joo Yoo* ${ }^{\dagger 1}$, Dushanthi Pinnaduwage ${ }^{\dagger 1}$, Daryl Waggott ${ }^{1}$, \\ Shelley B Bull ${ }^{1,2}$ and Lei Sun ${ }^{2,3}$
}

Addresses: ${ }^{1}$ Samuel Lunenfeld Research Institute of Mount Sinai Hospital, 600 University Avenue, Toronto, Ontario M5G 1X5 Canada,
${ }^{2}$ Dalla Lana School of Public Health, University of Toronto, 6th Floor, Health Sciences Building, 155 College Street, Toronto, Ontario M5T 3M7
Canada and ${ }^{3}$ Department of Statistics, University of Toronto, 100 St. George Street, Toronto, Ontario M5S 3G3 Canada

E-mail: Yun Joo Yoo* - yoo@lunenfeld.ca; Dushanthi Pinnaduwage - pinnad@lunenfeld.ca; Daryl Waggott - waggott@lunenfeld.ca; Shelley B Bull - bull@lunenfeld.ca; Lei Sun - sun@utstat.toronto.edu

*Corresponding author †Equal contributors

from Genetic Analysis Workshop 16

St Louis, MO, USA 17-20 September 2009

Published: 15 December 2009

BMC Proceedings 2009, 3(Suppl 7):SI03 doi: 10.1186/1753-656I-3-S7-SI03

This article is available from: http://www.biomedcentral.com/I753-656I/3/S7/SI03

(c) 2009 Yoo et al; licensee BioMed Central Ltd.

This is an open access article distributed under the terms of the Creative Commons Attribution License (http://creativecommons.org/licenses/by/2.0), which permits unrestricted use, distribution, and reproduction in any medium, provided the original work is properly cited.

\begin{abstract}
The power of genome-wide association studies can be improved by incorporating information from previous study findings, for example, results of genome-wide linkage analyses. Weighted falsediscovery rate (FDR) control can incorporate genome-wide linkage scan results into the analysis of genome-wide association data by assigning single-nucleotide polymorphism (SNP) specific weights. Stratified FDR control can also be applied by stratifying the SNPs into high and low linkage strata. We applied these two FDR control methods to the data of North American Rheumatoid Arthritis Consortium (NARAC) study and the Framingham Heart Study (FHS), combining both association and linkage analysis results. For the NARAC study, we used linkage results from a previous genome scan of rheumatoid arthritis (RA) phenotype. For the FHS study, we obtained genome-wide linkage scores from the same $550 \mathrm{k}$ SNP data used for the association analyses of three lipids phenotypes (HDL, LDL, TG). We confirmed some genes previously reported for association with RA and lipid phenotypes. Stratified and weighted FDR methods appear to give improved ranks to some of the replicated SNPs for the RA data, suggesting linkage scan results could provide useful information to improve genome-wide association studies.
\end{abstract}

\section{Background}

Use of prior or additional information may improve the power of single-nucleotide polymorphism (SNP)-disease association analysis. In particular, genome-wide linkage scans can provide complementary information to genome-wide association studies (GWAS). The weighted false-discovery rate control (WFDR) method incorporates genome-wide linkage study results by converting 
the linkage scores into SNP-specific weights then rescaling the association $p$-value for each SNP [1]. The stratified FDR control method (SFDR [2]) prioritizes genomic regions according to the available linkage scores. WFDR requires the investigator to choose and assign weights to SNPs whereas in SFDR, stratum-specific weights are internally derived by the choice of strata and the distribution of data [3]. SFDR is designed to use prior information to assign SNPs into strata that are more or less likely to include true-positive associations, which can similarly improve the power of GWAS, but is more robust than WFDR to uninformative or even misleading prior information [3]. We applied these two FDR methods along with the original FDR method to the North American Rheumatoid Arthritis Consortium (NARAC) study data provided for Genetic Analysis Workshop 16 (GAW 16) using previously reported linkage study results for rheumatoid arthritis (RA) [4]. We also performed genome-wide linkage and association analyses of the FHS data and applied the three FDR methods. We compared the regions of association identified by the different methods.

\section{Methods}

\section{Samples, phenotypes, and genotypes}

\section{RA data}

The NARAC data provided for GAW 16 included a set of 868 cases and 1,194 controls with information on a binary outcome (RA affection status) and on 545,080 genome-wide SNP genotypes from the Illumina $550 \mathrm{k}$ chip as well as the physical locations for the SNPs. In our association analysis, RA affection status was defined as positive for anti-cyclic citrinullated peptide antibody (anti-CCP).

\section{FHS data}

SNP genotyping data were provided based on the GeneChip Human Mapping $500 \mathrm{k}$ Array and $50 \mathrm{k}$ Human Gene Focused Panel. We analyzed a combined sample of the Offspring Cohort $\left(N_{1}=2,584\right)$ and the Generation 3 Cohort $\left(N_{2}=3,811\right)$ for association with each of the blood lipid measures, high-density lipoprotein (HDL), low-density lipoprotein (LDL), and triglyceride (TG), and included all family members in the sample who had been genotyped and phenotyped. The mean of lipid measures over multiple exams was adjusted for age, sex, body mass index (BMI), alcohol intake, and cigarette smoking. The phenotype measures of treated people were imputed using the methods in Kathiresan et al. [5] and Levy et al. [6].

\section{Quality control of SNP data}

We excluded SNPs with Hardy-Weinberg equilibrium $p$-value $\leq 10^{-9}$ in controls, missing genotype rate $>5 \%$, and minor allele frequency $<0.01$. Samples were also filtered by individual call missing rate $>5 \%$, duplicity, and relatedness. Within autosomes, there were 490,915 SNPs remaining in the RA data and 430,292 SNPs in the FHS data after applying this set of quality control criteria, using the computer program PLINK [7].

\section{Genome-wide association analysis}

RA data

Each SNP was tested for association using the 1-df allelic chi-squared test assuming an additive genotype model, implemented in PLINK [7].

\section{FHS data}

SNP association was evaluated using adjusted residual mean values obtained from the generalized estimating equation model for familial correlation. We split families unconnected in the Offspring and Generation 3 Cohort using the R package "kinship" [8]. Generalized estimating equation fitting was performed using a SAS GENMOD procedure assuming an exchangeable working correlation matrix.

\section{Genome-wide linkage analysis \\ RA data}

Results of the NARAC linkage study of RA using 642 Caucasian families and high-density SNP genotyping, as reported in Amos et al. [4], were used as the available prior linkage information, based on RA status (anti-CCP positivity) as the phenotype. The linkage scores at SNP markers across the genome, publicly available as supplementary information, were the nonparametric linkage (NPL) scores computed by Amos et al. [4] using linkage disequilibrium (LD) eliminated SNP genotypes. For the chromosomes with large centromeres $(1,3,9,11,16$, and 19), they assumed zero recombination of the centromeric regions.

\section{FHS data}

We performed a genome-wide linkage scan for each of the three lipid phenotypes values (i.e., covariate adjusted residuals), using 8,545 individuals from 1,349 FHS families $(3,928$ founders, 4,617 non-founders; 4,363 females and 4,182 males; family size ranging from 3 to 19). We selected 5,102 SNPs for the linkage scan according to the criteria of MAF $>0.45$, HWE test $p$-values in founders $>0.05$, individual genotype missing rate $<5 \%$, SNP missing rate $<2 \%$, mendelian error rate $<5 \%$, and LD measure $r^{2}<0.05$ between SNPs. Genomewide linkage scans were performed using the regression methods of MERLIN-REGRESS (version 1.1.2) [9,10]: the identical-by-descent (IBD) allele-sharing status for all relative pairs was regressed on the squared differences and squared sums of the pairs' trait values. This method 
requires specification of the population trait mean, variance, and heritability. We therefore estimated the heritability using the variance-components (VC) option in MERLIN. Lacking an available genetic map, we interpolated the deCODE map from the Affymetrix, Inc. website for the 5,102 selected SNPs.

\section{SNP-specific linkage scores}

The linkage score corresponding to each of the $\sim 550 \mathrm{k}$ GWA SNPs, $Z_{i}, i=1, \ldots, M$, was interpolated from the linkage scores of the available neighboring markers according to the relative distance between markers.

\section{False-discovery rate control methods}

False-discovery rate (FDR) control was performed by computing $q$-values using the method suggested by Storey [11]. If the $q$-value of a single SNP analysis was less than the chosen FDR control threshold value, the hypothesis of no association between the SNP and the disease was rejected.

\section{Stratified FDR}

Based on the linkage scan results, high and low linkage regions were determined using a NPL threshold value of $C=1.64$ for the NARAC RA data and LOD threshold value $C=0.5$ for the FHS data. SNPs that fell into a highlinkage region were grouped as Stratum $1\left(Z_{i} \geq C\right)$ and SNPs that fell into a low-linkage region were grouped as
Stratum $2\left(Z_{i}<C\right)$. FDR control was applied separately for the SNPs in Stratum 1 and Stratum 2 [11].

\section{Weighted FDR}

The weight of each SNP was obtained as $w_{i}=\exp \left(B \cdot Z_{i}\right) /$ $v$, where $v=\sum_{i}^{M} \exp \left(B \cdot Z_{i}\right) / M$ and $B=1$ (exponential weighting [1]). FDR was applied to weighted p-values, $p_{j} / w_{j}$, and the corresponding $q$-values were computed.

\section{Results}

\section{Results of the RA data analysis}

The SNPs in chromosome 6 (MHC region) showed very strong association with $p$-values less than $10^{-100}$ and also very high linkage scores (NPL $>16)$. To focus on results outside regions of established importance, Table 1 excludes chromosome 6 SNPs and presents results of SNPs with ranks $\leq 10$ based on any of FDR methods or SNPs from genes previously reported to be associated with RA [12-14]. Most of the latter were ranked higher than other SNPs in the genome. For some SNPs, mostly in the stronger linkage regions, either SFDR or WFDR improved the rank more than the other. For example, the original rank of rs1018361 in CTLA4 was 285 using FDR, which changed to 28 and 96 using SFDR and WFDR, respectively. In some weak linkage regions (TRAF1, WDFY4), WFDR retained similar ranks as FDR, whereas the SFDR ranks increased. However, $q$-values for WFDR were generally much higher than those of FDR and SFDR

Table I: Results of FDR, SFDR, and WFDR analyses of selected SNPs for the RA phenotype from the NARAC study

\begin{tabular}{|c|c|c|c|c|c|c|c|c|}
\hline \multirow[t]{2}{*}{$\mathbf{S N P}^{\mathrm{a}}$} & \multirow[t]{2}{*}{ Chr } & \multirow[t]{2}{*}{ BP } & \multirow[t]{2}{*}{ Gene } & \multirow{2}{*}{$\begin{array}{c}\text { Association } \\
\text { p-value }\end{array}$} & \multicolumn{3}{|c|}{ Rank } & \multirow{2}{*}{$\begin{array}{l}\text { Linkage } \\
\text { NPL score }\end{array}$} \\
\hline & & & & & FDR & SFDR & WFDR & \\
\hline rs668320I & 1 & 17426583 & PADI $4^{\mathrm{b}}$ & $1.32 \times 10^{-3}$ & 3531 & 4172 & 5320 & -0.11 \\
\hline rs247660I & I & 114089610 & PTPN22 $^{\mathrm{b}}$ & $2.91 \times 10^{-12}$ & I & 1 & 1 & 0.1 \\
\hline rs254294I & 2 & 172235228 & CYBRDI & $4.25 \times 10^{-6}$ & 69 & 5 & 36 & 1.76 \\
\hline rs6433309 & 2 & 172343658 & No gene & $1.50 \times 10^{-6}$ & 35 & 2 & 21 & 1.74 \\
\hline rs|3031008 & 2 & | $7830262 \mid$ & ТTCЗОА & $8.13 \times 10^{-6}$ & $|2|$ & 11 & 38 & 2.24 \\
\hline rs|269359| & 2 & 191686008 & STATI ${ }^{\mathrm{b}}$ & $3.98 \times 10^{-2}$ & 39083 & 15997 & 5028 & 3.38 \\
\hline rs6752770 & 2 & 191799069 & STAT4v & $7.28 \times 10^{-3}$ & 11404 & 2787 & 1554 & 3.42 \\
\hline rs 10184573 & 2 & 200273759 & No gene & $5.63 \times 10^{-6}$ & 84 & 6 & 22 & 2.93 \\
\hline rs 1018361 & 2 & $20451034 \mid$ & CTLA $4^{\mathrm{b}}$ & $3.36 \times 10^{-5}$ & 288 & 28 & 98 & 2.14 \\
\hline rs6596147 & 5 & 133075674 & No gene & $4.61 \times 10^{-9}$ & 3 & 8 & 4 & 0.16 \\
\hline rs6978820 & 7 & |46629802 & CNTNAP2 & $2.22 \times 10^{-6}$ & 46 & 3 & 24 & 1.79 \\
\hline rs 2900180 & 9 & 120785936 & $T R A F I^{\mathrm{b}}$ & $3.08 \times 10^{-9}$ & 2 & 7 & 3 & 0.01 \\
\hline rs7037673 & 9 & 120820038 & $C 5^{\mathrm{b}}$ & $2.15 \times 10^{-5}$ & 212 & 400 & 306 & 0.01 \\
\hline rs267।692 & 10 & 49767825 & WDFY4 & $2.66 \times 10^{-8}$ & 10 & 18 & 10 & 0.28 \\
\hline rsl|8253| & 20 & 57826397 & PHACTR3 & $4.83 \times 10^{-9}$ & 4 & 9 & 2 & 0.58 \\
\hline rs9974986 & 21 & 35262686 & $R U N X I^{\mathrm{b}}$ & $3.79 \times 10^{-3}$ & 7246 & 8165 & 6401 & 0.68 \\
\hline rs7।3756 & 22 & 43|| 8847 & No gene & $2.10 \times 10^{-8}$ & 9 & 17 & 8 & 0.08 \\
\hline
\end{tabular}

${ }^{\text {a}}$ The SNPs listed in the table include the most significant SNP from each of the previously reported genes and SNPs with ranks $\leq 10$ (one SNP per region) based on any of the FDR methods.

${ }^{b}$ Genes previously reported to be associated with RA.

${ }^{\mathrm{C}}$ Genome-wide linkage NPL scores $\geq 1.64$ (the chosen SFDR threshold) are in bold. 
(results not shown). These analyses suggest several new associations with RA (e.g., CNTNAP2 on chromosome 7).

\section{Results of the FHS data analysis}

Table 2 presents the results of SNPs selected with ranks $\leq$ 10 based on any of the three FDR methods or those most significant among genes previously reported for association with TG $[5,15]$. All SNPs with rank $\leq 10$ resided in the previously reported genes. SNPs in stronger linkage regions showed improvement in rank using SFDR and WFDR (linkage scores in bold). However, some of the gene regions previously reported for TG, HDL, or LDL were not confirmed in the FHS samples.

\section{Discussion}

The SFDR and WFDR methods can improve power of genome-wide association analyses when linkage scans are informative $[1,3]$. In the RA and FHS studies, using SFDR and WFDR improved ranks of SNPs in new and previously reported regions, suggesting improved power.

The threshold value for Stratum 1 and 2 in SFDR was somewhat arbitrarily set as 1.64 for the RA data (where the linkage results were NPL scores) and 0.5 for the FHS data (where the linkage results are LOD scores) to maintain the proportion of Stratum 1 to be about 5\% when the power of linkage scans is relatively low. The effect of small differences in threshold values for SFDR was insignificant on average in a simulation study [3]. In the RA and FHS data sets, the choice of different thresholds did produce some differences in ranking values, but the effect was minimal.

The power under FDR control depends on the proportion of true alternative hypotheses (true causal SNPs) in a family of tests. By preserving most of the potentially true causal SNPs in a small-sized Stratum 1, we can improve study power [3]. However, how to choose this threshold to optimize power remains an open question. A similar question applies to the choice of the weighting scheme (i.e., the value of the $B$ parameter) for the WFDR method.

SFDR and WFDR control methods using previous linkage results can be extended to multi-marker analysis with fixed or sliding windows, for example, by using the average linkage score within a window as a measure of prior information. Further study is warranted to evaluate extensions to multi-marker analysis settings.

\section{List of abbreviations used}

Anti-CCP: Anti-cyclic citrinullated peptide antibody; BMI: Body mass index; FDR: False-discovery rate; GAW: Genetic Analysis Workshop; GWAS: Genome-wide association studies; HDL: High-density lipoprotein; IBD: Identical by descent; LD: Linkage disequilibrium; LDL: Low-density lipoprotein; NARAC: North American Rheumatoid Arthritis Consortium; NPL: Nonparametric linkage; RA: Rheumatoid arthritis; SFDR: Stratified false-discovery rate control method; SNP: Single-nucleotide polymorphism; TG: Triglyceride; VC: Variance-component; WFDR: Weighted falsediscovery rate control.

Table 2: Results of FDR, SFDR, and WFDR analysis of selected SNPs for the TG phenotype from the FHS study

\begin{tabular}{|c|c|c|c|c|c|c|c|c|}
\hline \multirow[t]{2}{*}{ SNP ${ }^{a}$} & \multirow[t]{2}{*}{ Chr } & \multirow[t]{2}{*}{ BP } & \multirow[t]{2}{*}{ Gene $^{b}$} & \multirow{2}{*}{$\begin{array}{c}\text { Association } \\
\text { p-value }\end{array}$} & \multicolumn{3}{|c|}{ Rank } & \multirow{2}{*}{$\begin{array}{l}\text { Linkage } \\
\text { LOD score }^{c}\end{array}$} \\
\hline & & & & & FDR & SFDR & WFDR & \\
\hline rs435023I & 1 & 62695248 & DOCK7 & $2.39 \times 10^{-3}$ & 2376 & 2953 & 2755 & 0.11 \\
\hline rs4846918 & i & 228367209 & GALNT2 & $2.00 \times 10^{-2}$ & $|4| 7 \mid$ & 9915 & 10990 & 0.56 \\
\hline rs780094 & 2 & $2759474 \mid$ & GCKR & $2.83 \times 10^{-10}$ & 18 & 16 & 16 & 1.1 \\
\hline rs673I583 & 2 & 202269099 & ALS2 & $9.12 \times 10^{-2}$ & 52483 & 55094 & 66274 & 0 \\
\hline rs 16872759 & 4 & 22118656 & GPRI 25 & $6.90 \times 10^{-3}$ & 5805 & 6490 & 6951 & 0.05 \\
\hline rsII 78977 & 7 & 72494985 & $B A Z \mid B$ & $2.17 \times 10^{-12}$ & 10 & 12 & 10 & 0 \\
\hline rs|7|45738 & 7 & 72620810 & $T B L 2$ & $1.28 \times 10^{-10}$ & 15 & 19 & 18 & 0 \\
\hline rs $174|103|$ & 8 & 19896590 & $L P L$ & $1.23 \times 10^{-17}$ & 1 & 1 & 2 & 0.91 \\
\hline rs2980875 & 8 & 126550929 & $T R|B|$ & $1.74 \times 10^{-9}$ & 21 & 24 & 21 & 0.38 \\
\hline rs 6475522 & 9 & 2120917 & SMARCA2 & $1.62 \times 10^{-2}$ & $1|89|$ & 7794 & 5774 & 1.14 \\
\hline rs3750929 & 11 & 82259235 & $P R C P$ & $1.73 \times 10^{-1}$ & 92300 & 96678 & ||5272 & 0 \\
\hline rs6589566 & 11 & 116157633 & APOA5 & $1.71 \times 10^{-12}$ & 9 & II & 9 & 0.02 \\
\hline rs 948028 & 11 & 120149657 & GRIK4 & $1.80 \times 10^{-3}$ & 1899 & 2234 & 1986 & 0.23 \\
\hline$r s 2451214$ & 17 & 78308343 & $T B C D$ & $1.98 \times 10^{-1}$ & 103764 & $10850 \mid$ & 129319 & 0 \\
\hline rs 3813136 & 19 & 15452333 & PGLYRP2 & $7.23 \times 10^{-1}$ & 333987 & 276706 & 250668 & 0.51 \\
\hline$r s 2424295$ & 20 & 20165327 & C20orf26 & $2.30 \times 10^{-2}$ & 15945 & 17260 & 18677 & 0.09 \\
\hline
\end{tabular}

${ }^{\text {a}}$ The SNPs listed in the table include the most significant SNP from each of the previously reported genes and SNPs with ranks $\leq 10$ (one SNP per region) based on any of the FDR methods.

${ }^{b}$ All genes listed were previously reported to be associated with RA.

${ }^{\mathrm{c}}$ Genome-wide linkage LOD scores $\geq 0.5$ (the chosen SFDR threshold) are in bold. 


\section{Competing interests}

The authors declare that they have no competing interests.

\section{Authors' contributions}

YJY contributed to the design and writing of the paper; association analysis of FHS data; and SFDR and WFDR analysis of RA and FHS data. DP contributed to the design and writing of the paper, association analysis of RA, data and linkage analysis of FHS data. DW contributed association analysis of FHS data and QC analysis of RA and FHS data. SBB contributed to the critical revision of the paper for important content. LS contributed to the design of the paper and critical revision of the paper for important content.

\section{Acknowledgements}

The Genetic Analysis Workshops are supported by NIH grant ROI GM03 I575 from the National Institute of General Medical Sciences. This research was supported by research grants from the Canadian Institutes of Health Research (CIHR MOP-84287).

This article has been published as part of BMC Proceedings Volume 3 Supplement 7, 2009: Genetic Analysis Workshop 16. The full contents of the supplement are available online at http://www.biomedcentral.com/ $1753-656 \mid / 3$ ? issue $=$ S7.

\section{References}

I. Roeder K, Bacanu S, Wasserman L and Devlin B: Using linkage genome scans to improve power of association in genome scans. Am J Hum Genet 2006, 78:243-252.

2. Sun L, Craiu RV, Paterson $A D$ and Bull SB: Stratified false discovery control for large scale hypothesis testing with application to genome-wide association studies. Genet Epidemiol 2006, 28:352-367.

3. Yoo YJ, Bull SB, Paterson AD, Waggott D, The DCCT/EDIC Research Group and Sun L: Were genome-wide linkage studies a waste of time? Exploiting candidate regions within genome-wide association studies. Genet Epidemiol in press.

4. Amos Cl, Chen WV, Lee A, Li W, Kern M, Lundsten R, Batliwalla F, Wener M, Remmers E, Kastner DA, Criswell LA, Seldin MF and Gregersen PK: High-density SNP analysis of 642 Caucasian families with rheumatoid arthritis identifies two new linkage regions on IIpI2 and 2q33. Genes Immun 2006, 7:277-286.

5. Kathiresan S, Manning AK, Demissie S, D'Agostino RB, Surti A, Guiducci C, Gianniny L, Burtt NP, Melander O, Orho-Melander M, Arnett DK, Peloso GM, Ordovas JM and Cupples LA: A genomewide association study for blood lipid phenotypes in the Framingham Heart Study. BMC Med Genet 2007, 8(suppl I): SI7.

6. Levy D, DeStefano AL, Larson MG, O'Donnell CJ, Lifton RP, Gavras H, Cupples LA and Myers RH: Evidence for a gene influencing blood pressure on chromosome 17. Genome scan linkage results for longitudinal blood pressure phenotypes in subjects from the Framingham Heart Study. Hypertension 2000, 36:477-483.

7. Purcell S, Neale B, Todd-Brown K, Thomas L, Ferreira MA, Bender D, Maller J, Sklar P, de Bakker PI, Daly MJ and Sham PC: PLINK: a tool set for whole-genome association and population-based linkage analysis. Am J Hum Genet 2007, 8I:559-575.

8. Therneau TM: On Mixed Effect Cox Models, Sparse Matrices, And Modelling Data From Pedigrees -. http://mayoresearch. mayo.edu/mayo/research/biostat/upload/kinship.pdf.

9. Abecasis GR, Cherny SS, Cookson WO and Cardon LR: Merlinrapid analysis of dense genetic maps using sparse gene flow trees. Nat Genet 2002, 30:97-10I.
10. Sham PC, Purcell S, Cherny SS and Abecasis GR: Powerful regression-based quantitative-trait linkage analysis of general pedigrees. Am J Hum Genet 2002, 71:238-253.

II. Storey JD: A direct approach to false discovery rates. J $R$ Stat Soc B 2002, 64:479-498.

12. Remmers EF, Plenge RM, Lee AT, Graham RR, Hom G, Behrens TW, de Bakker PI, Le JM, Lee HS, Batliwalla F, Li W, Masters SL, Booty MG, Carulli JP, Padyukov L, Alfredsson L, Klareskog L, Chen WV, Amos Cl, Criswell LA, Seldin MF, Kastner DL and Gregersen PK: STAT4 and the risk of rheumatoid arthritis and systemic lupus erythematosus. N Engl J Med 2007, 357:977-986

13. Plenge RM, Seielstad M, Padyukov L, Lee AT, Remmers EF, Ding B, Liew A, Khalili H, Chandrasekaran A, Davies LR, Li W, Tan AK, Bonnard C, Ong RT, Thalamuthu A, Pettersson S, Liu C, Tian C, Chen WV, Carulli JP, Beckman EM, Altshuler D, Alfredsson L, Criswell LA, Amos Cl, Seldin MF, Kastner DL, Klareskog L and Gregersen PK: TRAFI-C5 as a risk locus for rheumatoid arthritis-a genomewide study. N Engl J Med 2007, 357: 1199-1209.

14. Begovich AB, Carlton VE, Honigberg LA, Schrodi SJ, Chokkalingam AP, Alexander HC, Ardlie KG, Huang Q, Smith AM, Spoerke JM, Conn MT, Chang M, Chang SY, Saiki RK, Catanese J], Leong DU, Garcia VE, McAllister LB, Jeffery DA, Lee AT, Batliwalla F Remmers E, Criswell LA, Seldin MF, Kastner DL, Amos CI, Sninsky Jj and Gregersen PK: A missence single-nucleotide polymorphism in a gene encodinga protein tyrosine phosphatise (PTPN22) is associated with rheumatoid arthritis. Am J Hum Genet 2004, 75:330-337.

15. Whole Genome Scan for Type 2 Diabetes in a Scandinavian Cohort. Related Metabolic Trait Results (updated May 2008) http:II www.broad.mit.eduldiabetes/scandinavs/metatraits.html.

Publish with Biomed Central and every scientist can read your work free of charge

"BioMed Central will be the most significant development for disseminating the results of biomedical research in our lifetime. "

Sir Paul Nurse, Cancer Research UK

Your research papers will be:

- available free of charge to the entire biomedical community

- peer reviewed and published immediately upon acceptance

- cited in PubMed and archived on PubMed Central

- yours - you keep the copyright 